OPEN ACCESS

Edited by:

Takeshi Sakurai,

University of Tsukuba, Japan

Reviewed by:

Yoshitaka Fukada,

The University of Tokyo, Japan

William Wisden,

Imperial College London,

United Kingdom

Arisa Hirano,

University of Tsukuba, Japan

*Correspondence:

Zhi-Li Huang

huangz@@fudan.edu.cn

${ }^{\dagger}$ These authors have contributed equally to this work

Specialty section

This article was submitted to Sleep and Circadian Rhythms,

a section of the journal

Frontiers in Neuroscience

Received: 07 August 2018

Accepted: 16 October 2018

Published: 05 November 2018

Citation:

Yuan X-S, Wei H-H, Xu W, Wang L,

Qu W-M, Li R-X and Huang Z-L (2018)

Whole-Brain Monosynaptic Afferent

Projections to the Cholecystokinin

Neurons of the Suprachiasmatic

Nucleus. Front. Neurosci. 12:807.

doi: 10.3389/fnins.2018.00807

\section{Whole-Brain Monosynaptic Afferent Projections to the Cholecystokinin Neurons of the Suprachiasmatic Nucleus}

\author{
Xiang-Shan Yuan ${ }^{\dagger}$, Hao-Hua Wei ${ }^{\dagger}$, Wei Xu, Lu Wang, Wei-Min Qu, Rui-Xi Li and \\ Zhi-Li Huang*
}

Department of Pharmacology, Department of Anatomy, School of Basic Medical Sciences, State Key Laboratory of Medical Neurobiology, Institutes of Brain Science and Collaborative Innovation Center for Brain Science, Fudan University, Shanghai, China

The suprachiasmatic nucleus (SCN) is the principal pacemaker driving the circadian rhythms of physiological behaviors. The SCN consists of distinct neurons expressing neuropeptides, including arginine vasopressin (AVP), vasoactive intestinal polypeptide (VIP), gastrin-releasing peptide (GRP), cholecystokinin (CCK), and so on. AVP, VIP, and GRP neurons receive light stimulation from the retina to synchronize endogenous circadian clocks with the solar day, whereas CCK neurons are not directly innervated by retinal ganglion cells and may be involved in the non-photic regulation of the circadian clock. To better understand the function of CCK neurons in non-photic circadian rhythm, it is vital to clarify the direct afferent inputs to CCK neurons in the SCN. Here, we utilized a recently developed rabies virus- and Cre/loxP-based, cell type-specific, retrograde tracing system to map and quantitatively analyze the whole-brain monosynaptic inputs to SCN CCK neurons. We found that SCN CCK neurons received direct inputs from 29 brain nuclei. Among these nuclei, paraventricular nucleus of the hypothalamus $(\mathrm{PVH})$, paraventricular nucleus of the thalamus (PVT), supraoptic nucleus (SON), ventromedial nucleus of the hypothalamus, and seven other nuclei sent numerous inputs to CCK neurons. Moderate inputs originated from the zona incerta, periventricular hypothalamic nucleus, and five other nuclei. A few inputs to CCK neurons originated from the orbital frontal cortex, prelimbic cortex, cingulate cortex, claustrum, and seven other nuclei. In addition, SCN CCK neurons were preferentially innervated by AVP neurons of the ipsilateral $\mathrm{PVH}$ and SON rather than their contralateral counterpart, whereas the contralateral PVT sent more projections to CCK neurons than to its ipsilateral counterpart. Taken together, these results expand our knowledge of the specific innervation to mouse SCN CCK neurons and provide an important indication for further investigations on the function of CCK neurons.

Keywords: cholecystokinin neuron, circadian rhythm, monosynaptic inputs, rabies viruses, suprachiasmatic nucleus 


\section{INTRODUCTION}

The suprachiasmatic nucleus (SCN) has been widely known as a central pacemaker that orchestrates physiological and behavioral circadian rhythms that need daily synchronization to stay in phase with the 24-h solar cycle. Light information is transmitted to the SCN by the retinohypothalamic tract (RHT), which is a monosynaptic projection from the retina to the SCN (Pickard, 1982; Moore et al., 2002; Hannibal and Fahrenkrug, 2006). The SCN is a small heterogeneous structure that contains diverse subpopulations of neurons expressing distinct neuropeptides, including arginine vasopressin (AVP), vasoactive intestinal polypeptide (VIP), gastrin-releasing peptide (GRP), calretinin, cholecystokinin (CCK), and so on (Abrahamson and Moore, 2001; LeSauter et al., 2002; Moore et al., 2002; Hannibal et al., 2010). The AVP, VIP, and GRP neurons in the SCN have been elucidated as retinorecipient neurons that receive direct inputs from the RHT, indicating that these neurons play an important role in mediating the effects of light on the circadian clock (Abrahamson and Moore, 2001; Antle and Silver, 2005; Fernandez et al., 2016). The CCK neuron was also identified as a distinct cell type in the SCN (Morin, 2013), but it did not receive projections from the RHT and was not activated by light (Hannibal et al., 2010). The circadian rhythm is principally synchronized by light, but it can also be regulated by non-photic signals, such as exercise, feeding, and temperature (Buxton et al., 2003; Escobar et al., 2009; Buhr et al., 2010; Burgess et al., 2010; Morris et al., 2012). Thus, the literature suggests that SCN CCK neurons may be involved in the nonphotic regulation of circadian rhythm. Previous studies have shown that feeding and temperature signals were mediated by the diencephalon, including the preoptic area, dorsomedial nucleus of the hypothalamus (DMH), arcuate nucleus of the hypothalamus (Arc), and paraventricular nucleus of the thalamus (PVT) (Tan et al., 2016; Hume et al., 2017; Zhao et al., 2017). However, it is not clear whether these nuclei send direct projections to CCK neurons of the SCN to entrain the circadian clock. Therefore, identifying the afferent circuits of SCN CCK neurons is critical to comprehensively understand the function of these neurons.

Traditional tracing approaches with non-specific tracers have successfully classified three major afferent inputs to the SCN: the RHT, geniculohypothalamic tract (GHT), and a pathway from the raphe nuclei (Hannibal and Fahrenkrug, 2006; Fernandez et al., 2016). However, a recent study used immunohistochemical staining to reveal that CCK neurons did not receive projections

\footnotetext{
Abbreviations: AH, anterior hypothalamic area; AI, agranular insular cortex; Arc, arcuate nucleus of the hypothalamus; BNST, bed nucleus of stria terminalis; $\mathrm{Cg}$, cingulate cortex; $\mathrm{DMH}$, dorsomedial nucleus of the hypothalamus; DR, dorsal raphe nucleus; IGL, intergeniculate leaf; LC, locus coeruleus; LH, lateral hypothalamic nucleus; LPO, lateral preoptic nucleus; LS, lateral septum; MnR, median raphe nucleus; MPO, medial preoptic nucleus; MO, motor cortex; OFC, orbital frontal cortex; PAG, periaqueductal gray; Prl, prelimbic cortex; PVH, paraventricular nucleus of the hypothalamus; PVT, paraventricular nucleus of the thalamus; RCh, retrochiasmatic area; SCN, suprachiasmatic nucleus; SON, supraoptic nucleus; SFO, subfomical organ; TC, tuber cinereum area; VDB, nucleus of the vertical limb of the diagonal band; $\mathrm{VMH}$, ventromedial nucleus of the hypothalamus; VMPO, ventromedial preoptic nucleus; ZI, zona incerta.
}

form these three major afferent pathways (Hannibal et al., 2010). The staining had obvious flaws in term of non-specificity and non-systematicity, making it difficult to assess the specific afferent distributions within the SCN. To overcome these limitations and detect all of the specific afferent inputs to SCN CCK neurons from the whole brain, it is necessary to adopt a specific, viral-mediated tracing system.

In recent years, a genetically modified rabies virus combined with Cre-LoxP technology, has been used to trace monosynaptic inputs (Wickersham et al., 2007; Lerner et al., 2015) and characterize the whole-brain presynaptic neurons of a specific neuron type within a complicated neural network (Do et al., 2016; Hu et al., 2016; Su et al., 2018). In our study, we utilized this viral tracing system to map the whole-brain afferent inputs to SCN CCK neurons. We found 29 afferent brain nuclei, including several important nuclei that integrated circadian, ingestive, and osmotic information to SCN CCK neurons. Our quantitative results provide numerous evidence for the structural framework of SCN CCK neurons and can guide further investigations of neuronal pathways that mediate functions of CCK neurons in the SCN.

\section{MATERIALS AND METHODS}

\section{Animals}

Pathogen-free, adult CCK-ires-Cre mice (Taniguchi et al., 2011) of either sex (10-12 weeks, 25-28 g) on a C57BL/6J background and wild-type littermates were used in these experiments. CCKires-Cre mice express Cre recombinase under the control of the CCK gene promoter. The animals were housed in individual cages at constant temperature $\left(22 \pm 0.5^{\circ} \mathrm{C}\right)$ and relative humidity $(60 \pm 2 \%)$ on an automatically controlled 12:12 light:dark cycle (lights on at 7 a.m.; 100 lux intensity) (Zhang et al., 2017) with free access to food and water. All of the animal studies were performed in accordance with protocols approved by the Committee on the Ethics of Animal Experiments of Fudan University Shanghai Medical College (permit number: 20110307049). Every effort was made to minimize the number of animals used as well as any pain or discomfort experienced by the subjects.

\section{Viruses and Surgery}

AAV-CAG-DIO-TVA-GFP (Adeno-associated virus (AAV) 2/9 serotype; titer $1.7 \times 10^{13}$ genomic copies/ml), AAV-CAG-DIORG (AAV2/9 serotype; titer $6.8 \times 10^{12}$ genomic copies $/ \mathrm{ml}$ ), and EnvA-pseudotyped, glycoprotein (RG)-deleted, dsRedexpressing rabies virus (RV-EvnA-DG-dsRed; RV $5.0 \times 10^{8}$ genomic copies $/ \mathrm{ml}$ ) were packaged and provided by BrainVTA (Wuhan, China). The detailed production and concentration procedures for the modified rabies virus were conducted as previously described (Pollak Dorocic et al., 2014; Hu et al., 2016).

Surgical procedures were carried out according to previous studies (Hu et al., 2016; Yuan et al., 2017). Briefly, naïve mice were anesthetized with chloral hydrate $(360 \mathrm{mg} / \mathrm{kg}$ ) and placed in a stereotaxic apparatus (RWD Life Science, Shenzhen, China). After exposing the skull and drilling a small hole, a glass micropipette was placed above the $S C N[0.5 \mathrm{~mm}$ posterior 
and $0.2 \mathrm{~mm}$ lateral from bregma; $5.1 \mathrm{~mm}$ ventral from the pial surface]. First, two helper AAVs (AAV-CAG-DIO-TVA-GFP and AAV-CAG-DIO-RG were mixed at a $1: 1$ ratio in $50 \mathrm{~nL}$ ) were injected into the $\mathrm{SCN}$ at $0.01 \mu \mathrm{L} / \mathrm{min}$ using a microsyringe pump controller (WPI, Sarasota, FL). To allow diffusion of the virus, the micropipette was not retracted until $15 \mathrm{~min}$ after the end of the injection. Three weeks later, $100 \mathrm{~nL}$ RV-EnvA-DGdsRed was similarly injected into the same site. The scalp wound was closed with surgical sutures, and each mouse was kept in a warm environment until it resumed normal activity as previously described (Luo et al., 2018b).

\section{Single-Cell RT-PCR}

At 1-2 weeks after only helper AAV injections, CCK-Cre mice were anesthetized and perfused transcardially with icecold modified aCSF saturated with $95 \% \mathrm{O}_{2}$ and $5 \% \mathrm{CO}_{2}$ and containing (in $\mathrm{mM}$ ): 215 sucrose, $26 \mathrm{NaHCO}_{3}, 10$ glucose, $3 \mathrm{MgSO}_{4}, 2.5 \mathrm{KCl}, 1.25 \mathrm{NaH}_{2} \mathrm{PO}_{4}, 0.6 \mathrm{mM}$ Na-pyruvate, 0.4 ascorbic acid, and $0.1 \mathrm{CaCl}_{2}$. Brains were then rapidly removed, and acute coronal slices $(300 \mu \mathrm{m})$ containing the SCN were cut on a vibratome (VT1200, Leica) in ice-cold modified aCSF. Next, slices were transferred to a holding chamber containing normal recording aCSF (in $\mathrm{mM}$ ): $125 \mathrm{NaCl}, 26 \mathrm{NaHCO}_{3}, 25$ glucose, 2.5 $\mathrm{KCl}, 2 \mathrm{CaCl}_{2}, 1.25 \mathrm{NaH}_{2} \mathrm{PO}_{4}$ and $1.0 \mathrm{MgSO}_{4}$, and allowed to recover for $30 \mathrm{~min}$ at $32^{\circ} \mathrm{C}$. Then, slices were maintained at room temperature (RT) for 30 min before recording.

During recording, slices were submerged in a recording chamber superfused with aCSF $(2 \mathrm{~mL} / \mathrm{min})$ at $30-32^{\circ} \mathrm{C}$. Slices were visualized using a fixed-stage upright microscope (BX51W1, Olympus, Japan) equipped with a $40 \times$ water immersion objective and an infrared-sensitive CCD camera. The CCK neurons were identified based on their GFP expression in the SCN and the cytosolic content was aspirated into the patch pipette, and expelled into a $200 \mu \mathrm{L}$ PCR tube as described previously (Luo et al., 2018b). The single-cell reverse-transcription PCR (RTPCR) protocol was designed to detect the presence of mRNA coding for CCK. Reverse transcription and PCR amplification were performed with gene-specific multiplex primer using the SuperScript III One-Step RT-PCR kit (catalog number: 12574018, ThermoFisher). The reaction was performed as follows: $30 \mathrm{~min}$ at $55^{\circ} \mathrm{C}, 2 \mathrm{~min}$ at $94^{\circ} \mathrm{C} ; 70$ cycles of $20 \mathrm{~s}$ at $94^{\circ} \mathrm{C}, 30 \mathrm{~s}$ at $61^{\circ} \mathrm{C}$, and $25 \mathrm{~s}$ at $68^{\circ} \mathrm{C}$; and $5 \mathrm{~min}$ at $68^{\circ} \mathrm{C}$. The PCR products were visualized by electrophoresis in agarose gels (1.5\%) with ethidium bromide. The expected size of each final PCR product is CCK 215 bp. The specific primers for CCK gene were custom designed and synthesized (Biosune Biotechnology, Shanghai). CCK-F primer, $5^{\prime}$ to $3^{\prime}$ : AAGCCATGAAGAGCGGCGTAT; CCK-R primer, $5^{\prime}$ to 3': GCGGACCTGCTGGATGTATCG.

\section{Histology and Image Analysis}

One week after injection of the rabies virus, mice were perfused with $10 \mathrm{~mL}$ saline, followed by $100 \mathrm{~mL}$ of $4 \%$ paraformaldehyde in $0.1 \mathrm{M}$ phosphate buffer ( $\mathrm{PB}, \mathrm{pH} 7.4)$. The brains were removed, post-fixed for $4-6 \mathrm{~h}$ at $4^{\circ} \mathrm{C}$, and then cryoprotected in 10,20, and $30 \%$ sucrose in $0.1 \mathrm{M} \mathrm{PB}$ at $4^{\circ} \mathrm{C}$ until they sank. Tissues were embedded in OCT compound, and stored at $-80^{\circ} \mathrm{C}$ before use. The brains were coronally sectioned at a thickness of $30 \mu \mathrm{m}$ on a cryostat (Leica 1950) in three series and were collected in $0.01 \mathrm{M}$ phosphate-buffered saline (PBS, $\mathrm{pH} 7.4$ ). Every third section was counterstained with DAPI (1:3000, Sigma-Aldrich, USA). The stained sections were then coverslipped with Fluoromount-G ${ }^{\mathrm{TM}}$ (Southern Biotech).

Images of whole-brain sections were captured using a $20 \times$ objective on an Olympus microscope (Olympus VS-120, Tokyo, Japan). Further imaging analyses were performed using Olympus analysis software and ImageJ software. The number of afferent neurons and area of each input regions were counted by ImageJ automatically, and we normalized the data in every input region. Quantification of the subregion boundaries was based on the mouse brain atlas of Paxinos and Franklin (Paxinos and Franklin, 2001). The proportion of cell in each nucleus was calculated as the ratio of the normalized number of dsRed-labeled cells in each nucleus to the total number of dsRed-labeled cells, and the cell density was defined as the number of dsRed-labeled cells per unit area within each nucleus. According to the proportion of cells in each nucleus, we defined three grades of afferent inputs as numerous input (over 4\%), moderate input (1-4\%), and a few input $(<1 \%)$.

To characterize the inputs from the paraventricular nucleus of the hypothalamus (PVH), and supraoptic nucleus (SON), we immunostained the brain slices containing the two areas with AVP and oxytocin (Oxt) antibodies according to the following protocol (Yuan et al., 2017). Brain sections from the $\mathrm{PVH}$ and SON were incubated overnight at $4^{\circ} \mathrm{C}$ in $\mathrm{PBS}$ containing 5\% normal donkey serum (v/v), 0.3\% Triton X$100(\mathrm{v} / \mathrm{v})$, and the following primary antibodies: rabbit antiAVP (1:2000, catalog number: 20069, Immunostar) and rabbit anti-Oxt (1:2000, catalog number: ab212193, Abcam). After several washes in PBS, the sections were incubated with Alexa Fluor-conjugated IgG antibody (Invitrogen) at room temperature for $2 \mathrm{~h}$. The sections were then incubated in PBS containing DAPI. Finally, sections were coverslipped with Fluoromount$\mathrm{G}^{\mathrm{TM}}$ (Southern Biotech). Fluorescence images were collected using a Leica confocal system.

\section{RESULTS}

\section{Mapping Monosynaptic Inputs Onto SCN CCK Neurons Using a Rabies-Based Tracing System}

To identify the monosynaptic afferent inputs to SCN CCK neurons, we used rabies virus-mediated, trans-synaptic, retrograde tracing on a transgenic mouse line expressing Cre recombinase in CCK neurons (Taniguchi et al., 2011). This retrograde, viral tracing system has been shown to label monosynaptic inputs to the desired starter cells with high specificity (Lerner et al., 2015; Do et al., 2016; Hu et al., 2016). In CCK-Cre mice, we applied a genetically engineered viral system to map the whole-brain afferent inputs to SCN CCK neurons. We first co-expressed the avian receptor TVA and the rabies glycoprotein G (RG) in SCN CCK neurons, which was achieved by a unilateral injection of two AAV-DIO helper viruses (AAV-CAG-DIO-TVA-GFP and AAV-CAG-DIO-RG) 
into the SCN (Figure 1A) of CCK-Cre mice. Three weeks later, RV-EnvA-DG-dsRed was injected into the same location, where it only infected cells expressing TVA and required RG to spread retrogradely into presynaptic cells (Figures 1A-C). After 1 week, the starter neurons could be characterized by the co-expression of RV-EnvA-DG-dsRed and AAV-CAG-DIO-TVA-GFP. We used single-cell RT-PCR to detect the presence of CCK mRNA in GFP-positive neurons in the SCN ( 7 of $7, n=3$ CCK-Cre mice), and confirmed that these neurons are CCK-expressing neurons (Figures 1D,E). We found that the starter neurons were restricted to the ventral part of the rostral and middle SCN, ipsilateral to the injection site (Figures 1F,H). Moreover, we observed numerous neurons in the SCN that were dsRed-positive but did not express GFP, demonstrating the presence of direct, monosynaptic input from other types of SCN neurons to the CCK neurons (Figure 1H). Upon conducting the same injection protocol in naïve animals, we did not detect the expression of GFP or dsRed in these wild-type mice that did not express Cre recombinase (Figure 1G). Thus, this technique could be used to map the whole-brain, monosynaptic afferent inputs to SCN CCK neurons (Figure 1C).

\section{SCN CCK Neurons Received Direct Inputs From 29 Brain Nuclei}

To investigate the whole-brain monosynaptic input areas to SCN CCK neurons, we examined the serial coronal wholebrain sections (Figure 2). In CCK-Cre mice injected with the three viruses, those neurons expressing only dsRed were the monosynaptic inputs to SCN CCK neurons. The dsRedlabeled presynaptic neurons were observed in 29 brain nuclei throughout the telencephalon, diencephalon, and brainstem (Figure 2). Furthermore, we measured the number of labeled neurons and the labeling density in individual brain areas to more quantitatively describe the whole-brain distribution of afferent input to SCN CCK neurons. The locations of labeled neurons were determined using a standard mouse brain atlas (Paxinos and Franklin, 2001). To correct potential bias, the number of dsRed-labeled cells in each nucleus was further normalized by the number of starter neurons. A list of wholebrain inputs was generated for SCN CCK neurons (Figure 3), which consisted of 29 different nuclei. Among these, CCK neurons received numerous afferent projections from 11 nuclei: PVH, PVT, SON, ventromedial nucleus of the hypothalamus (VMH), Arc, ventromedial preoptic nucleus (VMPO), medial preoptic nucleus (MPO), tuber cinereum area (TC), DMH, anterior hypothalamic area $(\mathrm{AH})$, retrochiasmatic area $(\mathrm{RCh})$. In addition, we found seven nuclei with moderate input nuclei contained the zona incerta, periventricular nucleus of the hypothalamus, lateral septum (LS), bed nucleus of stria terminalis (BNST), lateral hypothalamus ( $\mathrm{LH})$, lateral preoptic nucleus (LPO), and the nucleus of the vertical limb of the diagonal band (VDB). Finally, we observed $11 \mathrm{a}$ few inputs to CCK neurons, which originated from the orbital frontal cortex (OFC), prelimbic cortex (Prl), cingulate cortex (Cg), claustrum, posterior hypothalamic area $(\mathrm{PH})$, dorsal raphe nucleus (DR), intergeniculate leaf (IGL), periaqueductal gray (PAG), locus coeruleus (LC), subfornical organ (SFO), and median raphe nucleus (MnR). Although most of the dsRed-labeled presynaptic neurons were located in the hemisphere ipsilateral to the starter neurons, the contralateral PVT had stronger projections to SCN CCK neurons than the ipsilateral PVT (712.4 \pm 565.9 vs. 410.5 \pm 304.5 cells $/ \mathrm{mm}^{2}$, Figure 4B). Furthermore, in comparison with previous studies, our study revealed five novel projections to CCK neurons, namely, the VDB, LPO, Prl, OFC, and Cg (Supplemental Figure 1) (Moga and Moore, 1997; Krout et al., 2002).

\section{Preferential Inputs to SCN CCK Neurons Were From Diencephalon, Not From the Three Major SCN Afferent Pathways}

We found that SCN CCK neurons integrated the majority of their inputs (91.97\%) from the diencephalon, specifically from the VMH (16.47\%), PVH (11.78\%), Arc (9.37\%), VMPO (7.66\%), PVT (6.16\%), MPO (6.23\%), and SON (4.67\%) (Figures 3, 4). Although the $\mathrm{VMH}$ provided the largest number of inputs to CCK neurons, the SON was the most densely labeled area (Figure 3). Interestingly, we detected no dsRed-labeled neurons in both ipsilateral and contralateral retina (data not shown), indicating that SCN CCK neurons did not receive direct input from the major photic input pathway, the RHT (Morin, 2013). Furthermore, we observed only sparse dsRed labeling in the IGL $(0.26 \%)$ and MnR (0.05\%) (Figures 3, 4F), which are part of the GHT and a serotonin pathway from the median raphe nuclei, respectively. In contrast with our results, these two major pathways were previously shown to send robust afferent inputs to the SCN (Morin, 2013). Our results also differed from the findings of Hannibal and colleagues, in which immunostaining did not reveal any direct innervation from the IGL and raphe nucleus to the CCK neurons of the SCN (Hannibal et al., 2010).

\section{SCN CCK Neurons Received Inputs From AVP Neurons in the PVH and SON}

The PVH and SON of the hypothalamus are two important integrative brain structures that coordinate responses to perturbations in water balance and regulate maternal physiology through the release of the neuropeptide hormones AVP and Oxt (Qiu et al., 2011). In this study, we found that while SCN CCK neurons received significant afferent inputs from bilateral PVH (Figures 2, 4E), the ipsilateral PVH had more projections to SCN CCK neurons than the contralateral PVH (2058.0 \pm 418.4 vs. $841.3 \pm 341.0$ cells $/ \mathrm{mm}^{2}, n=4$, Figures 5A,B). Immunostaining for AVP showed that many PVH AVP neurons sent direct inputs onto SCN CCK neurons, whereas Oxt immunostaining demonstrated that few PVH Oxt neurons sent direct projections to CCK neurons (Figures 5C,D). Moreover, our results revealed that bilateral $\mathrm{SON}$ sent direct projections to the CCK neurons in the SCN, with more monosynaptic inputs from the ipsilateral SON than the contralateral SON $\left(2598.7 \pm 369.5\right.$ vs. $645.6 \pm 104.1$ cells $\left./ \mathrm{mm}^{2}, n=4\right)$, similar to the afferent pattern from the PVH. The immunostaining data for AVP and Oxt in the SON showed that many SON AVP neurons sent direct inputs onto SCN CCK neurons, whereas few 
A

$$
\begin{array}{|l|}
\hline \text { AAV-CAG-DIO-TVA-GFP } \\
\hline \hline \text { AAV-CAG-DIO-RG }
\end{array}
$$

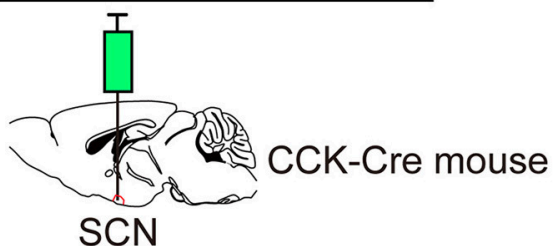

$\mathrm{SCN}$

Day 1

\section{RV-EnvA-DG-dsRed}

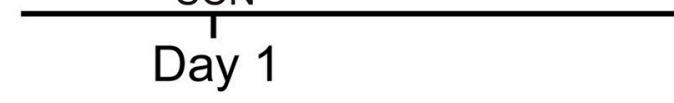

B

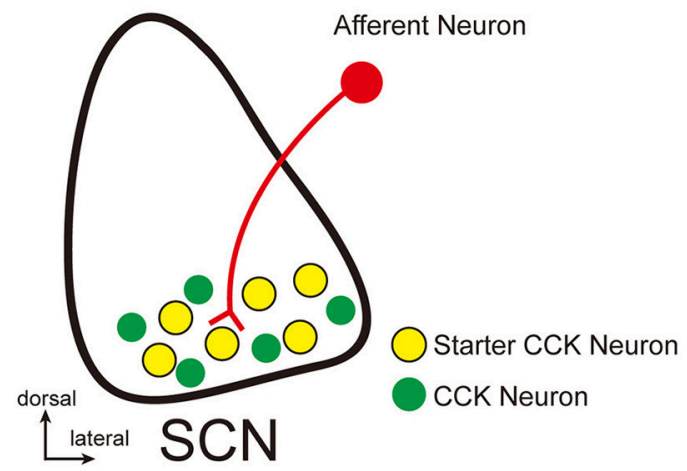

C
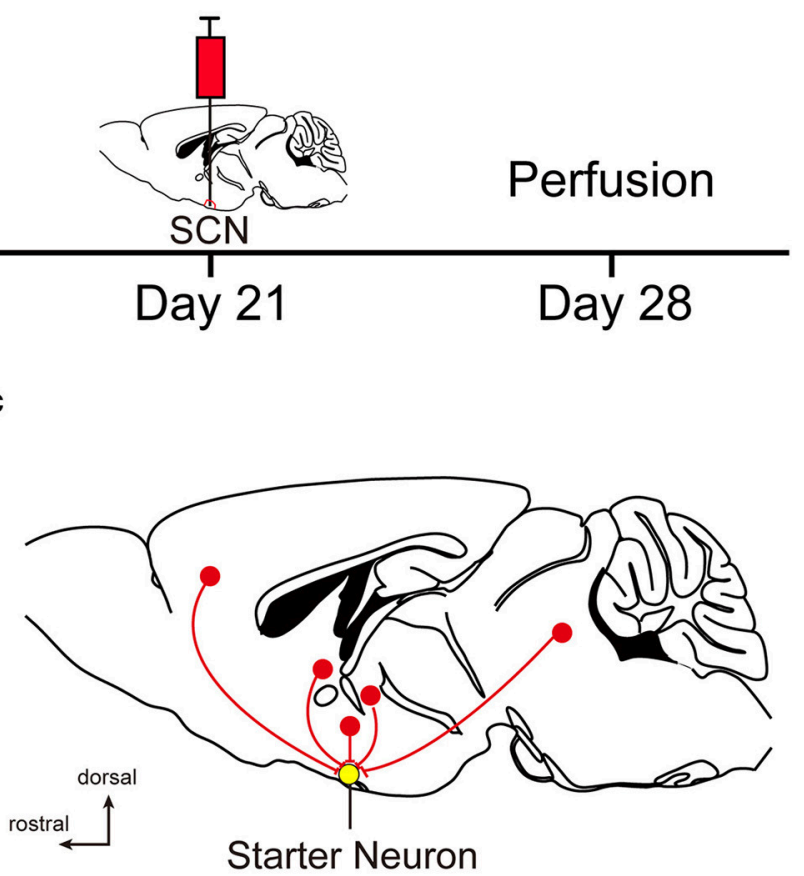

Starter Neuron
D

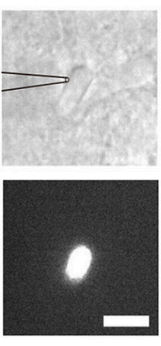

E

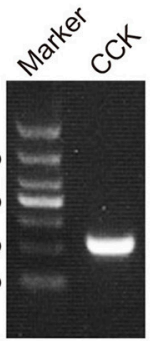

F
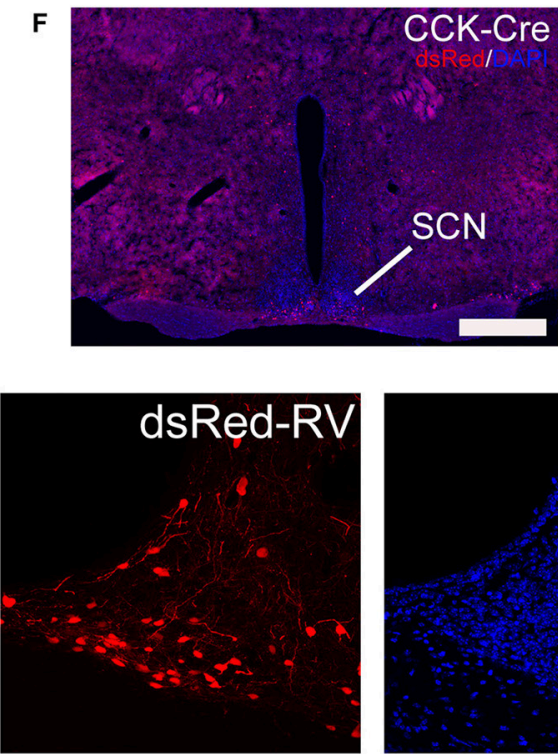

G
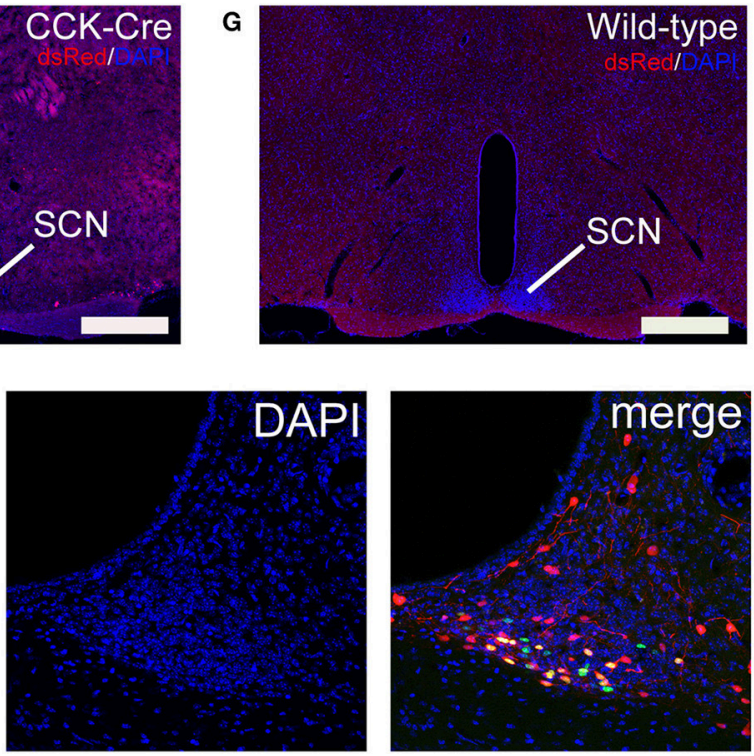

FIGURE 1 | Monosynaptic afferent tracing on SCN CCK neurons with a rabies virus-based, retrograde tracing system. (A) Design of viral vectors for RV-mediated trans-synaptic retrograde tracing and experimental timeline for unilateral injections of AAV and RV in the SCN of CCK-Cre mice. (B) Schematic illustration of the starter neuron (yellow, B) after AAV helper virus (green) and rabies virus (red) injection into SCN CCK neurons. (C) Schematic illustration of whole-brain, monosynaptic input (red) to CCK starter neurons (yellow). (D) A typical section of an CCK-Cre mouse injected with helper AAVs into the SCN for patch-clamp electrophysiology shows an GFP-expressing neuron for recording, the patch pipette attached to the membrane of the recorded neuron in phase contrast (upper panel), and the recorded neuron with fluorescent contrast (lower panel). Scale bar, $15 \mu \mathrm{m}$. (E) Representative result from a single-cell RT-PCR reaction confirming the CCK phenotype in the GFP labeled neuron of the SCN. (F,G) Fluorescence images showing RV-labeled neurons (red) in CCK-Cre mice (F), but not in wild-type mice (G). Scale bar, $500 \mu \mathrm{m}$. (H) Fluorescence images showing that starter neurons (yellow) infected with AAV helper virus and RV were restricted to the unilateral SCN. Scale bar, $50 \mu \mathrm{m}$. Data were obtained from independent experiments. 

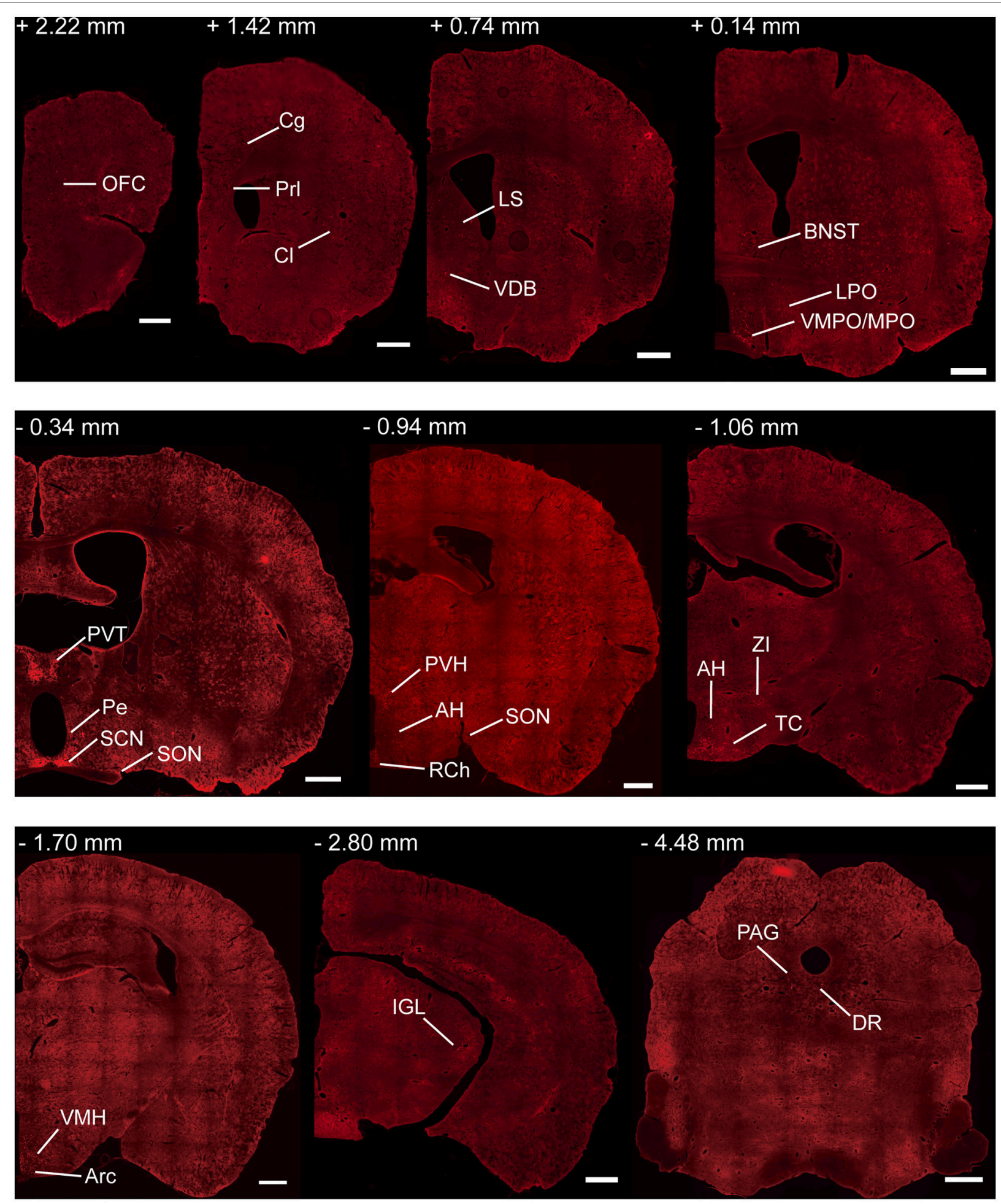

FIGURE 2 | Representative coronal sections showing labeling of monosynaptic inputs to SCN CCK neurons. For some sections, only one hemisphere is shown. Scale bar, $500 \mu \mathrm{m}$. Data were obtained from four independent experiments. Abbreviations of the brain regions used are the following: $\mathrm{AH}$, anterior hypothalamic area; Arc, arcuate nucleus of the hypothalamus; BNST, bed nucleus of stria terminalis; Cg, cingulate cortex; Cl, claustrum; DR, dorsal raphe nucleus; IGL, intergeniculate leaf; LPO, lateral preoptic nucleus; LS, lateral septum; MPO, medial preoptic nucleus; PAG, periaqueductal gray; Pe, periventricular nucleus of the hypothalamus; Prl, prelimbic cortex; PVH, paraventricular nucleus of the hypothalamus; PVT, paraventricular nucleus of the hypothalamus; RCh, retrochiasmatic area; SCN, suprachiasmatic nucleus; SON, supraoptic nucleus; TC, tuber cinereum area; VDB, nucleus of the vertical limb of the diagonal band; VMH, ventromedial nucleus of the hypothalamus; VMPO, ventromedial preoptic nucleus; ZI, zona incerta. 


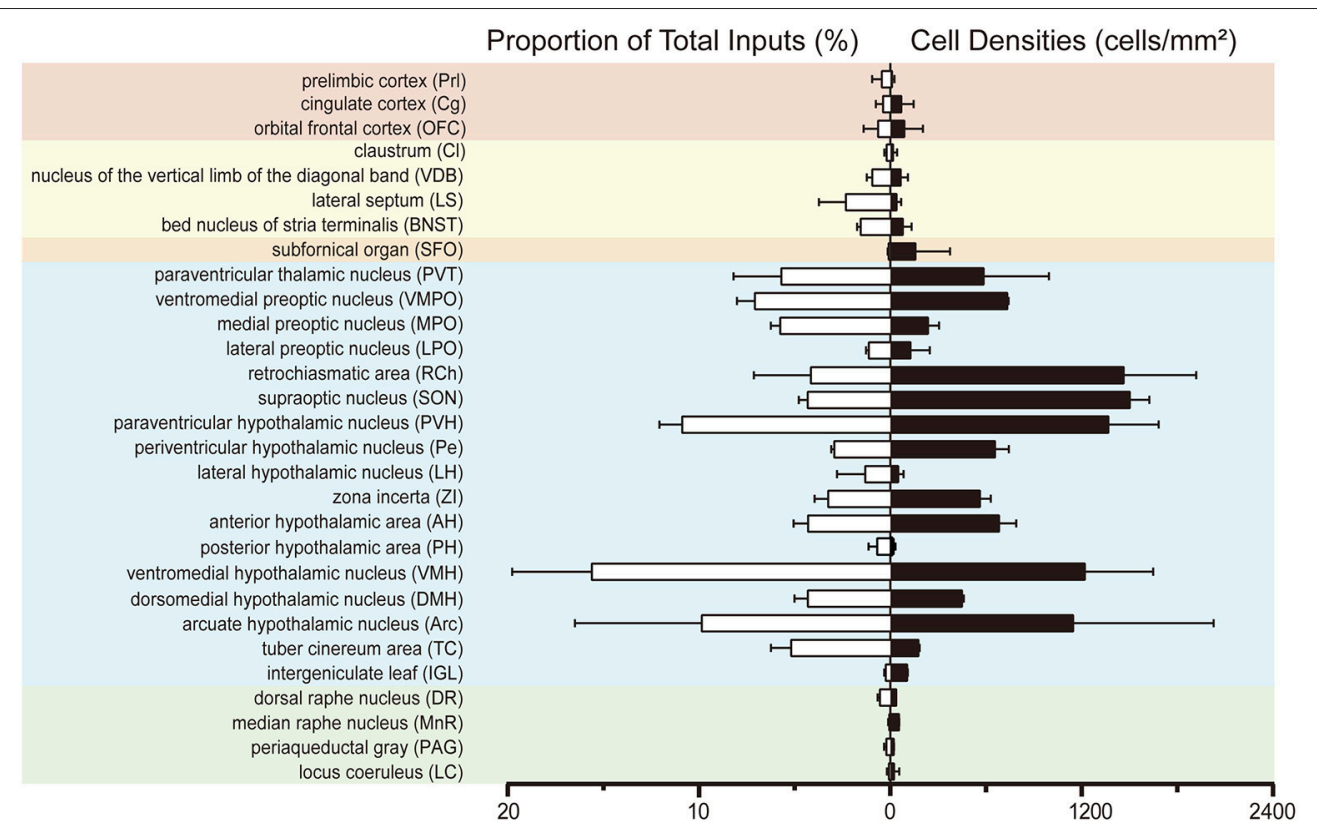

FIGURE 3 | Statistical analysis of whole-brain, monosynaptic inputs to SCN CCK neurons. Normalized ratio (Left) of cell number in each input region against the total number of whole-brain inputs. Cell densities (Right) of monosynaptic inputs in each brain area. Error bar represents the SEM ( $n=4$ CCK-Cre mice).

SON Oxt neurons sent direct projections to SCN CCK neurons (Figures 6A-D). These results can provide a foundations for further investigations on links between AVP- and Oxt-mediated ingestive behavior or osmotic stability and circadian rhythm in the SCN.

\section{DISCUSSION}

To understand how SCN CCK neurons modulate nonphotic behaviors, it is crucial to explore the afferent inputs that influence the activity of CCK neurons. In the present study, we clarified the whole-brain, direct, monosynaptic inputs to CCK neurons in the SCN using cell-type specific infection and retrograde spread of a modified rabies virus. We efficiently characterized the distribution of whole-brain input to SCN CCK neurons, which preferentially originated from a wide range of nuclei in the diencephalon, such as the VMH, Arc, MPO, PVT, PVH, SON, TC, and DMH. Moreover, the afferent pattern to CCK neurons in the SCN was strongly ipsilateral, with few contralateral projections other than the PVT. In addition, our results revealed specific inputs to the SCN and provided a comprehensive map of the presynaptic patterns that may control SCN CCK neuron activity.

\section{Comparison Between Specific Trans-Synaptic Tracing and Traditional Tracing}

The neural connectivity of the SCN has been extensively investigated due to its critical role in the circadian rhythm.
Previous investigations used conventional tracing techniques, such as non-specific tracers, multisynaptic pseudorabies viruses, and immunohistochemistry (Moga and Moore, 1997; Abrahamson and Moore, 2001; Krout et al., 2002; Morin, 2013; Fernandez et al., 2016). Previous tract-tracing studies have consistently revealed that the SCN received input from three major pathways: direct visual input from the retina through the RHT, secondary visual input from the IGL of the lateral geniculate complex through the GHT, and a pathway from the midbrain raphe nuclei (Hannibal and Fahrenkrug, 2006). However, these tracing methods do not allow for the identification of specific afferents of SCN CCK neurons. Using the rabies virus-based approach of trans-synaptic retrograde tracing, we found that the retina did not provide direct input to SCN CCK neurons. These results can explain why CCK neurons were not light-responsive as evaluated by the induction of c-Fos (Hannibal et al., 2010). Moreover, we revealed that SCN CCK neurons received minor projections from the IGL and raphe nuclei, unlike previous reports showing robust input projections from these brain nuclei (Krout et al., 2002) or no inputs at all (Hannibal and Fahrenkrug, 2006). In addition, we detected retrograde labeling in several brain areas, namely the Prl, OFC, Cg, VDB, and LPO, which had not been previously identified as SCN inputs. These novel observations were likely due to the greater sensitivity of the trans-synaptic tracing method using the modified rabies virus. Traditional tracttracing studies tended to inject small amounts of tracer into only a part of the $\mathrm{SCN}$, in an attempt to avoid the potential confounding factors of tracer spillover and tracer pickup by fibers-of-passage. Different research groups have reported inconsistent labeling patterns, especially from brain areas that 
A

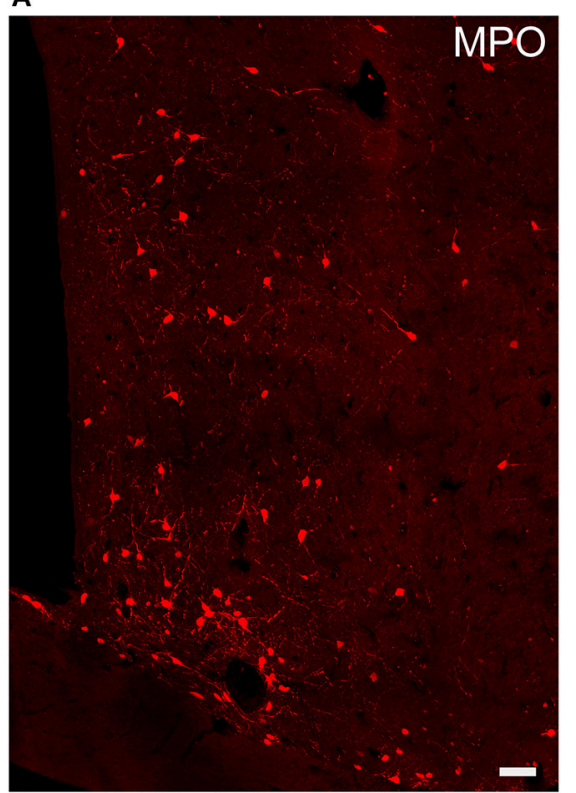

B

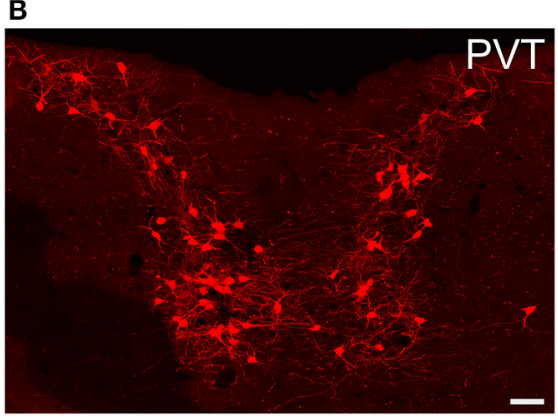

C

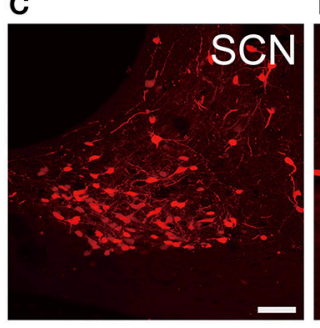

E
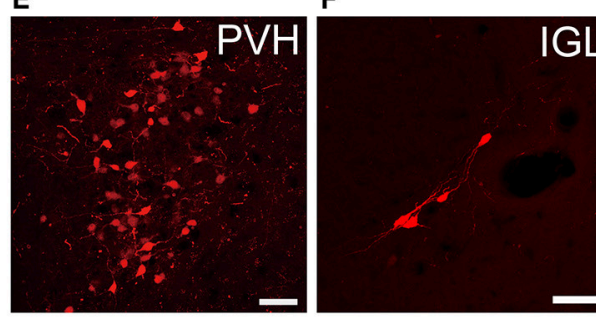

G

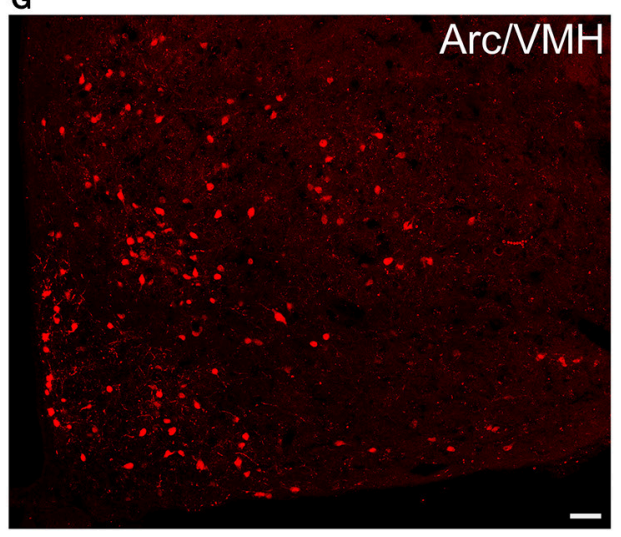

FIGURE 4 | Representative images of selected regions with monosynaptic inputs to SCN CCK neurons. Data were obtained from four independent experiments. (A) MPO; (B) PVT; (C) SCN; (D) SON; (E) PVH; (F) IGL; (G) Arc, and VMH. Scale bar, $50 \mu \mathrm{m}$.

contributed a few and moderate inputs to the SCN (Moga and Moore, 1997; Krout et al., 2002). Using the more precise and efficient viral-mediated tracing method, our findings should provide a comprehensive map of the presynaptic patterns that control SCN CCK neurons.

\section{Implications for SCN CCK Neurons in Non-photic Circadian Rhythm}

The SCN is widely considered to be the master circadian pacemaker necessary for physiological behaviors. Although light is the most potent zeitgeber to this master circadian oscillator, circadian clocks are entrained by both photic and non-photic signals. Previous studies found that AVP, VIP, and GRP neurons in the SCN received direct input from the retina, as well as indirect photic input from the IGL to integrate the non-imageforming photic information for circadian photo-entrainment (Abrahamson and Moore, 2001; Fernandez et al., 2016). As mentioned above, CCK neurons did not receive projections from the retina and were not directly entrained by light. However, our results revealed that there were robust dsRed-labeled neurons in the SCN, which did not co-express TVA-GFP, indicating that SCN CCK neurons received local innervations from intrinsic neurons in the SCN. It has been reported that SCN CCK neurons were innervated by the processes of SCN VIP neurons in mice and received contacts from SCN calbindin neurons in the ventral part of the central SCN in hamster (LeSauter et al., 2002, 2009; Hannibal et al., 2010). Therefore, SCN CCK neurons may relay optic or circadian signals from the VIP and calbindin neurons in the SCN and transmit these signals to mediate clock information.

Circadian entrainment can also occur in the absence of light, suggesting that non-photic signals can phase-shift and synchronize circadian clocks (Morris et al., 2012). Such non-photic signals include exercise, feeding, and temperature (McArthur et al., 1991; Damiola et al., 2000; Buxton et al., 2003; Escobar et al., 2009; Buhr et al., 2010). Previous findings showed that a majority of the preoptic neurons were activated by heat exposure and were located in the VMPO (Tan et al., 2016; Abbott and Saper, 2018). Our results revealed that the VMPO region had a robust projection to SCN CCK neurons; the interconnection 

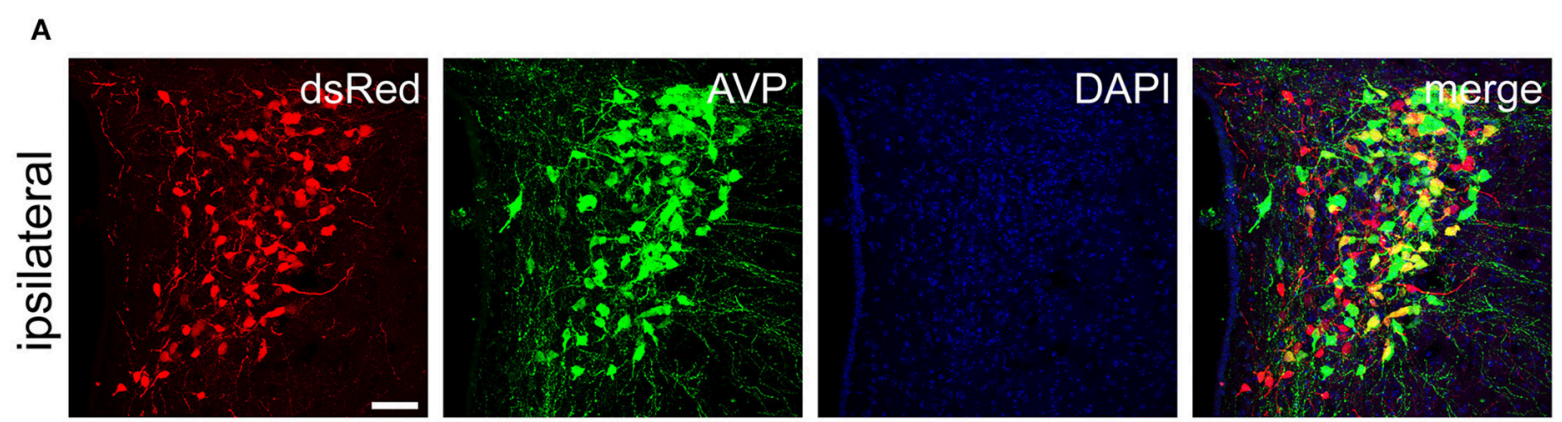

B
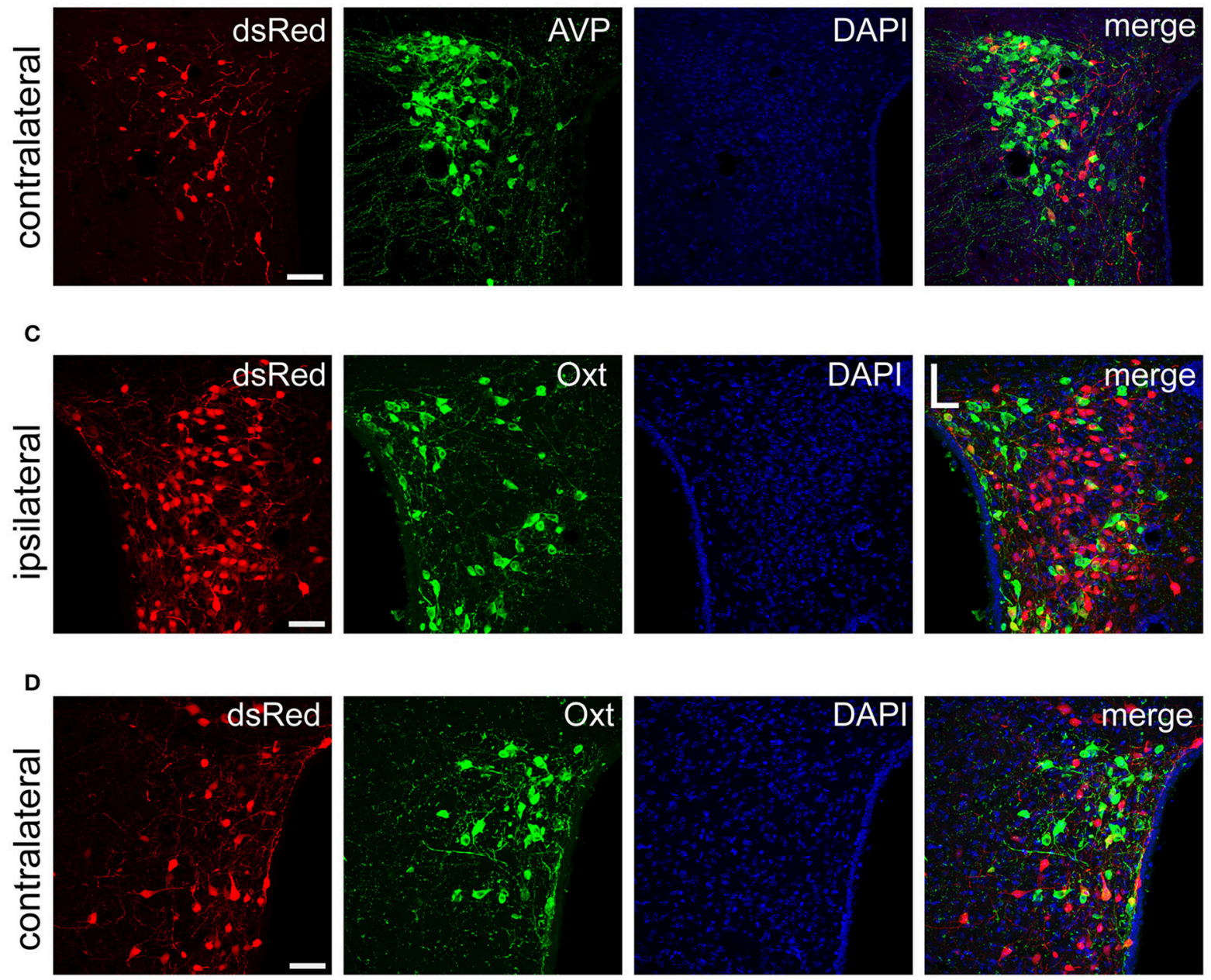

FIGURE 5 | Immunofluorescence images showing dsRed-labeled afferent neuron with AVP and Oxt in the bilateral PVH. Immunofluorescence images showing that some dsRed-labeled neurons were co-localized with AVP neurons in the ipsilateral (A) and the contralateral PVH (B). Immunofluorescence images showing that dsRed-labeled neurons rarely co-localized with Oxt neurons in the ipsilateral (C) and the contralateral PVH (D). Scale bar, $50 \mu \mathrm{m}$. Data were obtained from four independent experiments.

between the two areas supports the idea of circadian entrainment by temperature. Moreover, hypothalamic nuclei, including PVH, Arc, $\mathrm{DMH}, \mathrm{VMH}$, and the lateral hypothalamus, were shown to govern energy balance via both metabolic and behavioral responses (Myers and Olson, 2012). Particularly, the VMH and Arc were thought to play a role in controlling food intake and peripheral metabolism (Dhillon et al., 2006; Zhang and van den Pol, 2016). Here, we revealed that SCN CCK neurons received numerous afferent inputs from the $\mathrm{PVH}, \mathrm{VMH}$, and Arc; thus, our results may provide a circuit-based explanation of how feeding can entrain circadian rhythm via SCN CCK neurons. 

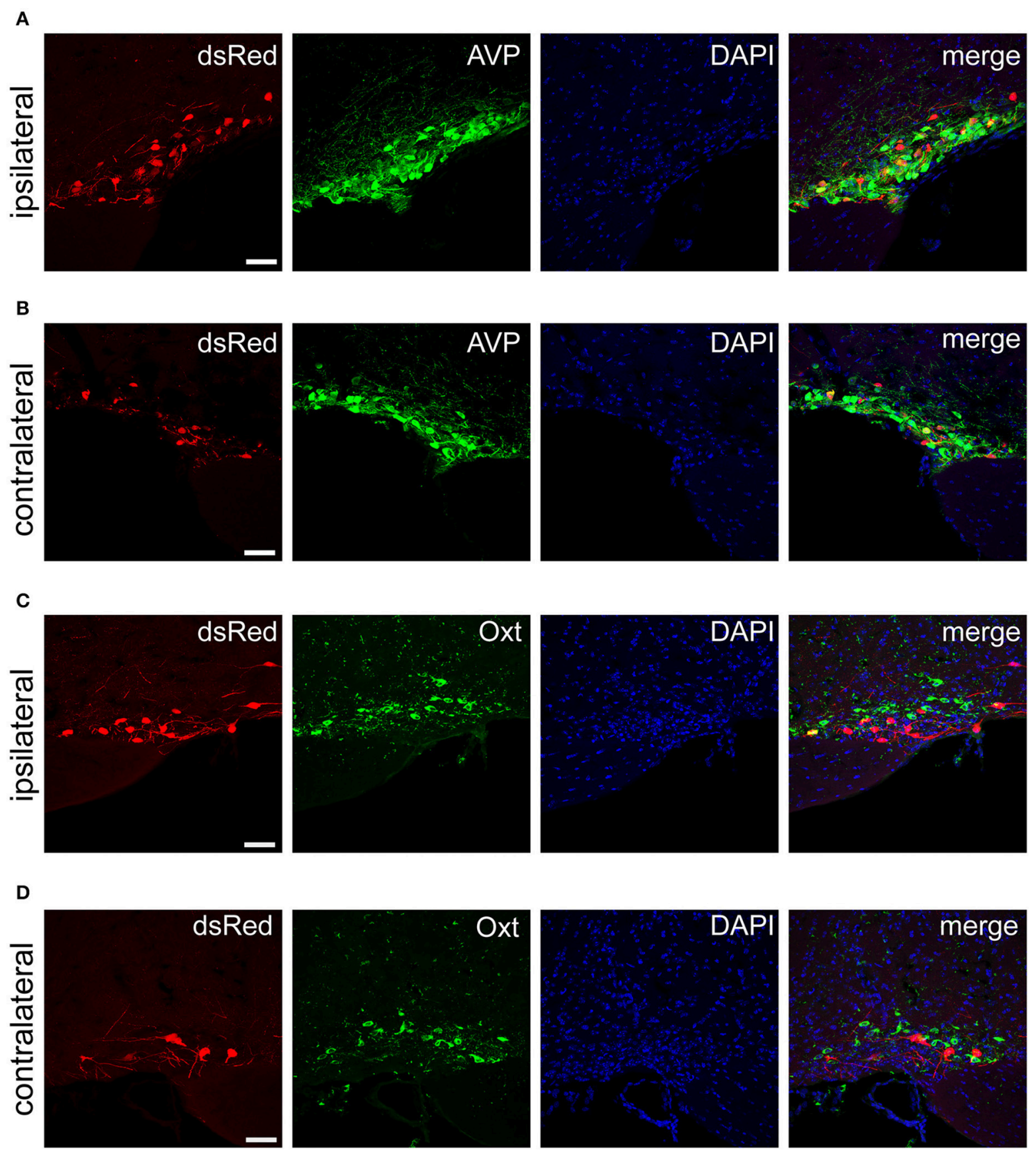

FIGURE 6 | Immunofluorescence images showing dsRed-labeled afferent neurons with AVP and Oxt in the bilateral SON. Immunofluorescence images showing that some dsRed-labeled neurons were co-localized with AVP neurons in the ipsilateral (A) and the contralateral SON (B). Immunofluorescence images showing that dsRed-labeled neurons rarely co-localized with Oxt neurons in the ipsilateral (C) and the contralateral SON (D). Scale bar, $50 \mu \mathrm{m}$. Data were obtained from four independent experiments.

\section{Implications for SCN CCK Neurons in Ingestive Behavior}

Ingestive behavior in a natural environment is essential for survival, and is dependent on hunger signals, which involve interoceptive sensory neurons that monitor metabolic level and consequently regulate food-seeking and consumption behaviors
(Trivedi, 2014; Cheng et al., 2018). Previous research has revealed many nuclei in the diencephalon as important sites that respond to feed restriction and regulate of ingestive behaviors, including the PVT, PVH, Arc, and VMH (Dhillon et al., 2006; Atasoy et al., 2012; Zhang and van den Pol, 2016; Jarvie et al., 2017; Ong et al., 2017; Luo et al., 2018a). Food deprivation incresed the 
synthesis of neuropeptide $\mathrm{Y}$, one of the most potent orexigenic peptides, in neurons of the Arc (Sahu et al., 1988; Beck et al., 1992), and acute and chronic calorie restriction increased the activity of Arc neurons (Jarvie et al., 2017). Moreover, neurons in the VMH were inhibited by food deprivation (Kosta et al., 1987; Sternson et al., 2005; Flanagan-Cato et al., 2008). For the PVT, the effect of food deprivation are controversial. Nakahara et al. (2004) observed a remarkable increase in c-Fos expression in the PVT after restricted feeding for $2 \mathrm{~h}$, suggesting increased neuronal activity by food deprivation. However, Zhang and van den Pol (2017) showed increased activity of ZI GABA neurons after food deprivation for $24 \mathrm{~h}$. Since ZI GABA neurons inhibit PVT glutamatergic neurons, the findings by Zhang and van den Pol suggest that the activity of PVT neurons was inhibited by food deprivation. Together, these data indicate that feed restriction can change the activity of neurons in the Arc, VMH, and PVT. The Arc receives the hunger signals via the neural circuits that integrate visceral signals of energetic state and consequently regulate physiology and behavior via efferent outputs of the Arc neurons, consisting of the agouti-related peptide (AGRP) neurons and pro-opiomelanocortin neurons. Our results showed that the Arc had abundant projections to SCN CCK neurons; this finding is consistent with previous investigations using nonspecific retrograde tracers that also showed that the SCN received major afferent inputs from the Arc. It has been speculated that the signal inputs from the Arc to CCK neurons could explain the circadian rhythm of feeding behavior, because CCK knockout mice eat more food than control animals during the light period and less food during the dark period (Lo et al., 2008). In addition, increasing evidence has shown that the PVT played a pivotal role in integrating information related to appetitive motivation, feeding, aversion, and anxiety (Hsu et al., 2014; Millan et al., 2017; Cheng et al., 2018). Our results, showing monosynaptic input from the PVT to SCN CCK neurons, suggest that CCK neurons could be involved in energy metabolism and anxietylike behavior (Lo et al., 2008). Interestingly, we observed a preferential input from the contralateral PVT to CCK neurons, in contrast to the primarily ipsilateral pattern of innervation from the Arc, PVH, and VMH. The function of this contralateraldominant innervation pattern between SCN CCK neurons and the PVT needs to be further studied.

\section{Implications for SCN CCK Neurons in Osmotic Stability}

Systemic osmoregulation is a vital homeostatic process because acute deviations in extracellular fluid osmolality can cause significant cellular shrinking or swelling and subsequent tissue and organ damage (Bedford and Leader, 1993). Thus, osmotic stability is controlled by centrally-mediated adjustments in the release of AVP from the hypothalamo-neurohypophyseal system. AVP is synthesized in the somata of magnocellular neurosecretory neurons located in the SON and $\mathrm{PVH}$, the crucial integrative brain structures that coordinate responses to perturbations in water balance (Bargmann, 1966; Qiu et al., 2011). It has been reported that the firing activity of neurons in the $\mathrm{PVH}$ and SON was enhanced during water deprivation
(Reis et al., 2012). Moreover, Landgraf and Ludwig (1991) have reported an increase in AVP release within the PVH and SON in response to local hypertonic artificial cerebrospinal fluid delivery. In addition, Miyata et al. (1994) have observed that the soma size of both AVP neurons in the SON in rat was enlarged in chronic osmotic stimulation. These data suggest that neurons in the PVH and SON, AVP-positive neurons, show response to osmotic disturbance.

In addition, in many species of mammals (Forsling, 2000; Moon et al., 2004), a progressive increase in circulating AVP concentration was observed during the sleep period and a trough around the wake period. This circadian rhythm in AVP levels is functionally important, because the absence of an AVP surge during the late-sleep stage resulted in polyuria and disrupted sleep (Miller, 2000). Recent findings revealed that clock neurons in the SCN sent direct, functional axonal projections to modulate the strength of the connection between the organum vasculosum lamina terminalis (the central osmosensory nucleus) and AVP neurons in the SON and PVH. This indicates that clock neurons can modulate circadian changes in osmotic and AVP regulation (Cui et al., 1997; Abrahamson and Moore, 2001; Kalsbeek et al., 2006; Trudel and Bourque, 2010, 2012). Our results suggest that SCN CCK neurons receive the osmotic signals from AVP neurons in the SON and PVH and may integrate the information of osmotic regulation with the principal pacemaker to generate the strongly reciprocal feedback circuit. When suffering from dehydration, this feedback circuit can help maintain circulating AVP at a predetermined level to exclude circadian fluctuations.

Notably, in addition to AVP expressing neurons, the $\mathrm{PVH}$ also contain another neuron populations, such as Oxt neurons in the magnocellular neuroendocrine group, neurons that synthesize and release corticotropin-releasing hormone, thyrotropinreleasing hormone, dopamine, somatostatin, or growth hormone-releasing hormone in the parvicellular neuroendocrine group, and neurons that project to the brainstem and spinal cord for the central autonomic regulation in the descending group (Kombian et al., 2002). In total, more than 30 neurotransmitters have been localized to neurons within the PVH (Pyner, 2009). Here, we found that a part of non-AVPand non-Oxt-positive neurons in the PVH also sent direct inputs to SCN CCK neurons, suggesting that SCN CCK neurons may have other functions in addition to osmotic regulation.

Together, our viral tracing results provide a whole-brain map of neurons that convey reinforcement signals to SCN CCK neurons. Our findings offer a new perspective for future explorations of circuit mechanisms mediating SCN functions, such as circadian rhythm, ingestive behavior, and osmotic stability. Therefore, it is critical to gather further functional and behavioral data on SCN CCK neurons.

\section{AUTHOR CONTRIBUTIONS}

$\mathrm{X}-\mathrm{SY}$ and H-HW designed and performed the experiments, analyzed data, and wrote the paper. WX performed the experiments. LW analyzed data and wrote the paper. W-MQ designed the experiments and analyzed data. R-XL and Z-LH 
designed the experiments, analyzed data, and wrote the paper.

\section{FUNDING}

This work was supported by the National Basic Research Program of China (Grant NO. 31530035, 81420108015 to Z-LH, Grant NO. 31671099, 31471064, 31871072 to W-MQ, and Grant NO. 31571103 to LW); the National Basic Research Program of China (Grant NO. 2015CB856401 to Z-LH).

\section{REFERENCES}

Abbott, S. B. G., and Saper, C. B. (2018). Role of the median preoptic nucleus in the autonomic response to heat-exposure. Temperature 5, 4-6. doi: $10.1080 / 23328940.2017 .1413155$

Abrahamson, E. E., and Moore, R. Y. (2001). Suprachiasmatic nucleus in the mouse: retinal innervation, intrinsic organization and efferent projections. Brain Res. 916, 172-191. doi: 10.1016/S0006-8993(01)02890-6

Antle, M. C., and Silver, R. (2005). Orchestrating time: arrangements of the brain circadian clock. Trends Neurosci. 28, 145-151. doi: 10.1016/j.tins.2005.01.003

Atasoy, D., Betley, J. N., Su, H. H., and Sternson, S. M. (2012). Deconstruction of a neural circuit for hunger. Nature 488, 172-177. doi: 10.1038/nature11270

Bargmann, W. (1966). Neurosecretion. Int. Rev. Cytol. 19, 183-201.

Beck, B., Burlet, A., Nicolas, J. P., and Burlet, C. (1992). Unexpected regulation of hypothalamic neuropeptide $\mathrm{Y}$ by food deprivation and refeeding in the Zucker rat. Life Sci. 50, 923-930.

Bedford, J. J., and Leader, J. P. (1993). Response of tissues of the rat to anisosmolality in-vivo. Am. J. Physiol. 264, R1164-R1179.

Buhr, E. D., Yoo, S. H., and Takahashi, J. S. (2010). Temperature as a universal resetting cue for mammalian circadian oscillators. Science 330, 379-385. doi: $10.1126 /$ science. 1195262

Burgess, H. J., Revell, V. L., Molina, T. A., and Eastman, C. I. (2010). Human phase response curves to three days of daily melatonin: $0.5 \mathrm{mg}$ versus $3.0 \mathrm{mg}$. J. Clin. Endocrinol. Metab. 95, 3325-3331. doi: 10.1210/jc.2009-2590

Buxton, O. M., Lee, C. W., L'Hermite-Baleriaux, M., Turek, F. W., and Van Cauter, E. (2003). Exercise elicits phase shifts and acute alterations of melatonin that vary with circadian phase. Am. J. Physiol. Regul. Integr. Comp. Physiol. 284, R714-R724. doi: 10.1152/ajpregu.00355.2002

Cheng, J., Wang, J., Ma, X., Ullah, R., Shen, Y., and Zhou, Y. D. (2018). Anterior paraventricular thalamus to nucleus accumbens projection is involved in feeding behavior in a novel environment. Front. Mol. Neurosci. 11:202. doi: 10.3389/fnmol.2018.00202

Cui, L. N., Saeb-Parsy, K., and Dyball, R. E. (1997). Neurones in the supraoptic nucleus of the rat are regulated by a projection from the suprachiasmatic nucleus. J. Physiol. 502 (Pt 1), 149-159.

Damiola, F., Le Minh, N., Preitner, N., Kornmann, B., Fleury-Olela, F., and Schibler, U. (2000). Restricted feeding uncouples circadian oscillators in peripheral tissues from the central pacemaker in the suprachiasmatic nucleus. Genes Dev. 14, 2950-2961. doi: 10.1101/gad.183500

Dhillon, H., Zigman, J. M., Ye, C., Lee, C. E., McGovern, R. A., Tang, V., et al. (2006). Leptin directly activates SF1 neurons in the VMH, and this action by leptin is required for normal body-weight homeostasis. Neuron 49, 191-203. doi: 10.1016/j.neuron.2005.12.021

Do, J. P., Xu, M., Lee, S. H., Chang, W. C., Zhang, S., Chung, S., et al. (2016). Cell type-specific long-range connections of basal forebrain circuit. Elife 5:e13214. doi: 10.7554/eLife.13214

Escobar, C., Cailotto, C., Angeles-Castellanos, M., Delgado, R. S., and Buijs, R. M. (2009). Peripheral oscillators: the driving force for food-anticipatory activity. Eur. J. Neurosci. 30, 1665-1675. doi: 10.1111/j.1460-9568.2009.06972.x

Fernandez, D. C., Chang, Y. T., Hattar, S., and Chen, S. K. (2016). Architecture of retinal projections to the central circadian pacemaker. Proc. Natl. Acad. Sci. U.S.A. 113, 6047-6052. doi: 10.1073/pnas.1523629113

\section{ACKNOWLEDGMENTS}

We thank Dr. Miao He (Fudan University) for kindly supplying us with CCK-ires-Cre mice.

\section{SUPPLEMENTARY MATERIAL}

The Supplementary Material for this article can be found online at: https://www.frontiersin.org/articles/10.3389/fnins. 2018.00807/full\#supplementary-material

Flanagan-Cato, L. M., Fluharty, S. J., Weinreb, E. B., and LaBelle, D. R. (2008). Food restriction alters neuronal morphology in the hypothalamic ventromedial nucleus of male rats. Endocrinology 149, 93-99. doi: 10.1210/en.2007-0008

Forsling, M. L. (2000). Diurnal rhythms in neurohypophysial function. Exp. Physiol. 85 Spec No, 179S-186S. doi: 10.1111/j.1469-445X.2000.tb00022.x

Hannibal, J., and Fahrenkrug, J. (2006). Neuronal input pathways to the brain's biological clock and their functional significance. Adv. Anat. Embryol. Cell Biol. 182, 1-71. doi: 10.1007/3-540-27789-7

Hannibal, J., Hundahl, C., Fahrenkrug, J., Rehfeld, J. F., and Friis-Hansen, L. (2010). Cholecystokinin (CCK)-expressing neurons in the suprachiasmatic nucleus: innervation, light responsiveness and entrainment in CCK-deficient mice. Eur. J. Neurosci. 32, 1006-1017. doi: 10.1111/j.1460-9568.2010.07385.x

Hsu, D. T., Kirouac, G. J., Zubieta, J. K., and Bhatnagar, S. (2014). Contributions of the paraventricular thalamic nucleus in the regulation of stress, motivation, and mood. Front. Behav. Neurosci. 8:73. doi: 10.3389/fnbeh.2014.00073

$\mathrm{Hu}, \mathrm{R}$., Jin, S., He, X., Xu, F., and $\mathrm{Hu}, \mathrm{J}$. (2016). Whole-brain monosynaptic afferent inputs to basal forebrain cholinergic system. Front. Neuroanat. 10:98. doi: $10.3389 /$ fnana.2016.00098

Hume, C., Sabatier, N., and Menzies, J. (2017). High-sugar, but not high-fat, food activates supraoptic nucleus neurons in the male rat. Endocrinology 158, 2200-2211. doi: 10.1210/en.2016-1640

Jarvie, B. C., King, C. M., Hughes, A. R., Dicken, M. S., Dennison, C. S., and Hentges, S. T. (2017). Caloric restriction selectively reduces the GABAergic phenotype of mouse hypothalamic proopiomelanocortin neurons. J. Physiol. 595, 571-582. doi: 10.1113/JP273020

Kalsbeek, A., Palm, I. F., La Fleur, S. E., Scheer, F. A., Perreau-Lenz, S., Ruiter, M., et al. (2006). SCN outputs and the hypothalamic balance of life. J. Biol. Rhythms 21, 458-469. doi: 10.1177/0748730406293854

Kombian, S. B., Hirasawa, M., Mouginot, D., and Pittman, Q. J. (2002). Modulation of synaptic transmission by oxytocin and vasopressin in the supraoptic nucleus. Prog. Brain Res. 139, 235-246. doi: 10.1016/S0079-6123(02)39020-4

Kosta, K., Kostova, D., Rehak, P., and Boda, K. (1987). Electrophysiological characteristic of the ventromedial hypothalamic nucleus and lateral hypothalamic area in normally fed and 24 hour food deprived conscious sheep. Arch. Tierernahr. 37, 1001-1007.

Krout, K. E., Kawano, J., Mettenleiter, T. C., and Loewy, A. D. (2002). CNS inputs to the suprachiasmatic nucleus of the rat. Neuroscience 110, 73-92. doi: 10.1016/S0306-4522(01)00551-6

Landgraf, R., and Ludwig, M. (1991). Vasopressin release within the supraoptic and paraventricular nuclei of the rat brain: osmotic stimulation via microdialysis. Brain Res. 558, 191-196.

Lerner, T. N., Shilyansky, C., Davidson, T. J., Evans, K. E., Beier, K. T., Zalocusky, K. A., et al. (2015). Intact-brain analyses reveal distinct information carried by SNc dopamine subcircuits. Cell 162, 635-647. doi: 10.1016/j.cell.2015.07.014

LeSauter, J., Bhuiyan, T., Shimazoe, T., and Silver, R. (2009). Circadian trafficking of calbindin-ir in fibers of SCN neurons. J. Biol. Rhythms 24, 488-496. doi: 10.1177/0748730409350876

LeSauter, J., Kriegsfeld, L. J., Hon, J., and Silver, R. (2002). Calbindin-D(28K) cells selectively contact intra-SCN neurons. Neuroscience 111, 575-585. doi: 10.1016/S0306-4522(01)00604-2

Lo, C. M., Samuelson, L. C., Chambers, J. B., King, A., Heiman, J., Jandacek, R. J., et al. (2008). Characterization of mice lacking the gene for 
cholecystokinin. Am. J. Physiol. Regul. Integr. Comp. Physiol. 294, R803-R810. doi: 10.1152/ajpregu.00682.2007

Luo, S. X., Huang, J., Li, Q., Mohammad, H., Lee, C. Y., Krishna, K., et al. (2018a). Regulation of feeding by somatostatin neurons in the tuberal nucleus. Science 361, 76-81. doi: 10.1126/science.aar4983

Luo, Y. J., Li, Y. D., Wang, L., Yang, S. R., Yuan, X. S., Wang, J., et al. (2018b). Nucleus accumbens controls wakefulness by a subpopulation of neurons expressing dopamine D1 receptors. Nat. Commun. 9:1576. doi: 10.1038/s41467-018-03889-3

McArthur, A. J., Gillette, M. U., and Prosser, R. A. (1991). Melatonin directly resets the rat suprachiasmatic circadian clock in vitro. Brain Res. 565, 158-161.

Millan, E. Z., Ong, Z., and McNally, G. P. (2017). Paraventricular thalamus: gateway to feeding, appetitive motivation, and drug addiction. Prog. Brain Res. 235, 113-137. doi: 10.1016/bs.pbr.2017.07.006

Miller, M. (2000). Nocturnal polyuria in older people: pathophysiology and clinical implications. J. Am. Geriatr. Soc. 48, 1321-1329. doi: 10.1111/j.1532-5415.2000.tb02608.x

Miyata, S., Itoh, T., Matsushima, O., Nakashima, T., and Kiyohara, T. (1994). Not only osmotic stress but also repeated restraint stress causes structural plasticity in the supraoptic nucleus of the rat hypothalamus. Brain Res. Bull. 33, 669-675.

Moga, M. M., and Moore, R. Y. (1997). Organization of neural inputs to the suprachiasmatic nucleus in the rat. J. Comp. Neurol. 389, 508-534.

Moon, D. G., Jin, M. H., Lee, J. G., Kim, J. J., Kim, M. G., and Cha, D. R. (2004). Antidiuretic hormone in elderly male patients with severe nocturia: a circadian study. BJU Int. 94, 571-575. doi: 10.1111/j.1464-410X.2004.05003.x

Moore, R. Y., Speh, J. C., and Leak, R. K. (2002). Suprachiasmatic nucleus organization. Cell Tissue Res. 309, 89-98. doi: 10.1007/s00441-002-0575-2

Morin, L. P. (2013). Neuroanatomy of the extended circadian rhythm system. Exp. Neurol. 243, 4-20. doi: 10.1016/j.expneurol.2012.06.026

Morris, C. J., Aeschbach, D., and Scheer, F. A. (2012). Circadian system, sleep and endocrinology. Mol. Cell. Endocrinol. 349, 91-104. doi: 10.1016/j.mce.2011.09.003

Myers, M. G., and Olson, D. P. (2012). Central nervous system control of metabolism. Nature 491, 357-363. doi: 10.1038/nature11705

Nakahara, K., Fukui, K., and Murakami, N. (2004). Involvement of thalamic paraventricular nucleus in the anticipatory reaction under food restriction in the rat. J. Vet. Med. Sci. 66, 1297-1300. doi: 10.1292/jvms.66.1297

Ong, Z. Y., Liu, J. J., Pang, Z. P., and Grill, H. J. (2017). Paraventricular thalamic control of food intake and reward: role of glucagon-like peptide-1 receptor signaling. Neuropsychopharmacology 42, 2387-2397. doi: $10.1038 /$ npp. 2017.150

Paxinos, G., and Franklin, K. B. J. (2001). The mouse brain in stereotaxic coordinates. San Diego, CA: Academic Press.

Pickard, G. E. (1982). The afferent connections of the suprachiasmatic nucleus of the golden hamster with emphasis on the retinohypothalamic projection. $J$. Comp. Neurol. 211, 65-83. doi: 10.1002/cne.902110107

Pollak Dorocic, I., Furth, D., Xuan, Y., Johansson, Y., Pozzi, L., Silberberg, G., et al. (2014). A whole-brain atlas of inputs to serotonergic neurons of the dorsal and median raphe nuclei. Neuron 83, 663-678. doi: 10.1016/j.neuron.2014. 07.002

Pyner, S. (2009). Neurochemistry of the paraventricular nucleus of the hypothalamus: implications for cardiovascular regulation. J. Chem. Neuroanat. 38, 197-208. doi: 10.1016/j.jchemneu.2009.03.005

Qiu, J., Hindmarch, C. C., Yao, S. T., Tasker, J. G., and Murphy, D. (2011). Transcriptomic analysis of the osmotic and reproductive remodeling of the female rat supraoptic nucleus. Endocrinology 152, 3483-3491. doi: 10.1210/en.2011-1044

Reis, W. L., Biancardi, V. C., Son, S., Antunes-Rodrigues, J., and Stern, J. E. (2012). Enhanced expression of heme oxygenase-1 and carbon monoxide excitatory effects in oxytocin and vasopressin neurones during water deprivation. $J$. Neuroendocrinol. 24, 653-663. doi: 10.1111/j.1365-2826.2011.02249.x

Sahu, A., Kalra, P. S., and Kalra, S. P. (1988). Food deprivation and ingestion induce reciprocal changes in neuropeptide $\mathrm{Y}$ concentrations in the paraventricular nucleus. Peptides $9,83-86$.

Sternson, S. M., Shepherd, G. M., and Friedman, J. M. (2005). Topographic mapping of $\mathrm{VMH}->$ arcuate nucleus microcircuits and their reorganization by fasting. Nat. Neurosci. 8, 1356-1363. doi: 10.1038/nn1550

Su, Y. T., Gu, M. Y., Chu, X., Feng, X., and Yu, Y. Q. (2018). Whole-brain mapping of direct inputs to and axonal projections from gabaergic neurons in the parafacial zone. Neurosci. Bull. 34, 485-496. doi: 10.1007/s12264-018-0216-8

Tan, C. L., Cooke, E. K., Leib, D. E., Lin, Y. C., Daly, G. E., Zimmerman, C. A., et al. (2016). Warm-sensitive neurons that control body temperature. Cell 167, 47-59 e15. doi: 10.1016/j.cell.2016.08.028

Taniguchi, H., He, M., Wu, P., Kim, S., Paik, R., Sugino, K., et al. (2011). A resource of Cre driver lines for genetic targeting of GABAergic neurons in cerebral cortex. Neuron 71, 995-1013. doi: 10.1016/j.neuron.2011.07.026

Trivedi, B. P. (2014). Neuroscience: dissecting appetite. Nature 508, S64-S65. doi: $10.1038 / 508$ S64a

Trudel, E., and Bourque, C. W. (2010). Central clock excites vasopressin neurons by waking osmosensory afferents during late sleep. Nat. Neurosci. 13, U467U488. doi: 10.1038/nn.2503

Trudel, E., and Bourque, C. W. (2012). Circadian modulation of osmoregulated firing in rat supraoptic nucleus neurones. J. Neuroendocrinol. 24, 577-586. doi: 10.1111/j.1365-2826.2012.02298.x

Wickersham, I. R., Lyon, D. C., Barnard, R. J., Mori, T., Finke, S., Conzelmann, K. K., et al. (2007). Monosynaptic restriction of transsynaptic tracing from single, genetically targeted neurons. Neuron 53, 639-647. doi: 10.1016/j.neuron.2007.01.033

Yuan, X. S., Wang, L., Dong, H., Qu, W. M., Yang, S. R., Cherasse, Y., et al. (2017). Striatal adenosine A2A receptor neurons control active-period sleep via parvalbumin neurons in external globus pallidus. Elife 6:e29055. doi: 10.7554/eLife.29055

Zhang, X., and van den Pol, A. N. (2016). Hypothalamic arcuate nucleus tyrosine hydroxylase neurons play orexigenic role in energy homeostasis. Nat. Neurosci. 19, 1341-1347. doi: 10.1038/nn.4372

Zhang, X., and van den Pol, A. N. (2017). Rapid binge-like eating and body weight gain driven by zona incerta GABA neuron activation. Science 356, 853-859. doi: 10.1126/science.aam7100

Zhang, Z., Wang, H.-J., Wang, D.-R., Qu, W.-M., and Huang, Z.-L. (2017). Red light at intensities above $10 \mathrm{~lx}$ alters sleep-wake behavior in mice. Light Sci. Appl. 6:e16231. doi: 10.1038/lsa.2016.231

Zhao, Z. D., Yang, W. Z., Gao, C., Fu, X., Zhang, W., Zhou, Q., et al. (2017). A hypothalamic circuit that controls body temperature. Proc. Natl. Acad. Sci. U.S.A. 114, 2042-2047. doi: 10.1073/pnas.1616255114

Conflict of Interest Statement: The authors declare that the research was conducted in the absence of any commercial or financial relationships that could be construed as a potential conflict of interest.

The reviewer $\mathrm{AH}$ and the handling Editor declared their shared affiliation, at the time of review.

Copyright (C) 2018 Yuan, Wei, Xu, Wang, Qu, Li and Huang. This is an open-access article distributed under the terms of the Creative Commons Attribution License (CC $B Y)$. The use, distribution or reproduction in other forums is permitted, provided the original author(s) and the copyright owner(s) are credited and that the original publication in this journal is cited, in accordance with accepted academic practice. No use, distribution or reproduction is permitted which does not comply with these terms. 